\title{
Dextran sulfate activates contact system and mediates arterial hypotension via $\mathrm{B}_{2}$ kinin receptors
}

\author{
MATTHIAS SIEBECK, JOHN C. CHERONIS, EDWIN FINK, JACEK KOHL, BERND SPIES, \\ MICHAEL SPANNAGL, MARIANNE JOCHUM, AND HANS FRITZ \\ Departments of Surgery, Medicine, and Clinical Chemistry and Clinical Biochemistry, Ludwig-Maximilians \\ University, Klinikum Innenstadt, D-80336 Munich, Germany
}

\begin{abstract}
Siebeck, Matthias, John C. Cheronis, Edwin Fink, Jacek Kohl, Bernd Spies, Michael Spannagl, Marianne Jochum, and Hans Fritz. Dextran sulfate activates contact system and mediates arterial hypotension via $B_{2}$ kinin receptors. J. Appl. Physiol. 77(6): 2675-2680, 1994.-To define some of the mechanisms underlying dextran sulfate (DXS)-induced hypotension, we investigated the effects of either the plasma kallikrein inhibitor des-Pro ${ }^{2}-\left[\mathrm{Arg}^{15}\right]$ aprotinin (BAY x 4620) or the specific bradykinin $B_{2}$-receptor antagonist Hoe-140 on the hypotensive response to DXS. In the first study, anesthetized miniature pigs were given DXS alone, DXS plus BAY x 4620 in various doses, or saline. As expected, DXS alone produced a profound but transient systemic arterial hypotension with a concomitant reduction in kininogen. Circulating kinin levels, complement fragment des-Arg-C3a, and fibrin monomer were all increased. Treatment with BAY x 4620 produced a dose-dependent attenuation of these effects with complete blockade of the hypotension as well as the observed biochemical changes at the highest dose $(360 \mathrm{mg})$. In a second study, two groups of pigs were given either DXS alone or DXS plus Hoe-140. DXS-induced hypotension was completely blocked by Hoe-140 pretreatment; however, kininogen was again depleted. We conclude, therefore, that DXS-induced hypotension is produced by activation of plasma kallikrein that results in the production of bradykinin and that liberation of bradykinin and its action on $B_{2}$ receptors in the vasculature are both necessary and sufficient to produce the observed effects on circulatory pressure.
\end{abstract}

plasma kallikrein inhibitor; pigs; serine protease

DEXTRAN SULFATE (DXS) is a negatively charged macromolecule that has been shown to activate prekallikrein (15). As such, it is one of a variety of compounds that are able to activate the contact phase of blood coagulation, a multifunctional system of proteases that controls vasoregulation (via the formation of the potent vasodilator bradykinin) and amplifies the humoral inflammatory response (via activation of the coagulation, fibrinolysis, and complement pathways) (2).

The essential components of the contact system are the zymogen factor XII (Hageman factor), a negatively charged surface or macromolecule that induces a change in the conformation of factor XII, high-molecular-mass kininogen, prekallikrein, and factor XI. In this complex, factor XII and prekallikrein can activate each other in the presence of kininogen, resulting in the cleavage of kininogen by kallikrein and the release of bradykinin (2). In addition, factor XIIa converts factor XI to its active form, factor XIa, thereby activating the coagulation cascade. Both the Hageman factor fragment (8) and thrombin (13) are able to activate the classical pathway, whereas plasma kallikrein can activate the alternative pathway (6) of the complement system. Interaction of plasma kallikrein, factor XIa, and factor XIIa (3) also leads to activation of single-chain urokinase-type plasminogen activator and of a contact system-dependent plasminogen proactivator (1). In their activated form, these proteases convert plasminogen to plasmin and are considered to be the primary agents involved in intrinsic fibrinolysis. Finally, bradykinin and thrombin induce the release of tissue-type plasminogen activator from endothelial cells, thereby triggering extrinsic fibrinolysis.

Because the contact system combines the activation of the plasma cascade systems with the release of the hypotensive mediator bradykinin, the system has long been implicated in the pathophysiology of septic shock, disseminated intravascular coagulation, and multiple organ failure including adult respiratory distress syndrome (14, 17). In addition, bacterial lipopolysaccharide has been shown to activate the contact system in vitro (18), and in humans plasma kallikrein has been shown to be activated during endotoxemia (5). Finally, plasma kallikrein inhibitors such as aprotinin (22) or $\alpha_{1}$-antitrypsin Pittsburgh (4) can attenuate the effects of bacterial- or endotoxin-induced experimental shock.

In a previous investigation we found that infusion of DXS decreased the amount of uncleaved kininogen (kinin-containing kininogen) in pig plasma and reduced systemic arterial pressure. Blood pressure returned to normal only after kinin-containing kininogen had been depleted. Both the cleavage of kininogen and hypotension were attenuated or blocked by $\mathrm{C}_{1}$-esterase inhibitor and high doses of aprotinin (9), both of which inhibit plasma kallikrein, suggesting a role for bradykinin in this effect. In rabbits, however, DXS-induced arterial hypotension has been shown to be mediated via the release of serotonin from platelets (25), which can be stimulated by thrombin, another mediator of the activated contact system.

The present investigation was conducted to clarify the mechanism of DXS-induced hypotension in pigs. It was our hypothesis that DXS-induced hypotension is primarily mediated by bradykinin and that other mediators such as serotonin, platelet-activating factor, and metabolites of arachidonic acid, if involved, are dependent on bradykinin. Two studies were performed, one with the plasma kallikrein inhibitor des-Pro ${ }^{2}$-[Arg $\left.{ }^{15}\right]$ aprotinin (BAY x 4620) (7) and the second with the dekapeptide D-Arg $\left[\mathrm{Hyp}^{3}, \mathrm{Thi}^{5}{ }^{5} \mathrm{D}^{\mathrm{T}} \mathrm{Tic}^{7}, \mathrm{Oic}^{8}\right]$ bradykinin (Hoe-140), a bradykinin $\mathrm{B}_{2}$-receptor antagonist (20). Both compounds were able to completely block DXS-induced hypotension, whereas only the former compound prevented the decrease of uncleaved kininogen. 


\section{MATERIALS AND METHODS}

\section{$D X S$ and $B A Y \times 4620$}

Recombinant BAY x 4620 (7) was generously provided by $P$. Stadler, D. Hörlein, and F. Schumann (Bayer AG, Wuppertal, Germany). For these studies, the compound was dissolved in sterile physiological saline and infused intravenously.

DXS, with a molecular mass of $\sim 500,000 \mathrm{Da}$ (Pharmacia LKB, Uppsala, Sweden), was dissolved in $30 \mathrm{ml}$ of sterile physiological saline $1 \mathrm{~h}$ before use and infused intravenously in a dose of $5 \mathrm{mg} / \mathrm{kg}$ for $1 \mathrm{~h}$. The dose of DXS used in this study was based on previous experimentation (9). Animals were observed for $2 \mathrm{~h}$ from the start of the DXS infusion.

On the basis of preliminary pharmacokinetic experiments (data not shown), the entire dose administered was divided into a bolus dose (one-third of the total) injected $15 \mathrm{~min}$ before the start of the DXS infusion, followed by a continuous infusion over 135 min during which time the remaining two-thirds of the dose was delivered. The total dose of BAY x 4620 was varied from 45 to $360 \mathrm{mg}$. Three control groups, 1) DXS plus saline, 2) saline alone, and 3) high-dose BAY x 4620 (360 mg) alone, were also included in this study.

All animal procedures were approved by the Regierung von Oberbayern and complied with the Tierschutzgesetz in der Fassung of August 18, 1986. The animals, 35 miniature pigs, were fasted overnight but had free access to water. Sedation was achieved by azaperone ( $3 \mathrm{mg} / \mathrm{kg} \mathrm{im})$ and metomidate $(3.75 \mathrm{mg} /$ $\mathrm{kg}$ ip). After induction of anesthesia with pentobarbital sodium ( $24 \mathrm{mg} / \mathrm{kg}$ iv), a $6.5-\mathrm{mm}$ orotracheal tube was placed and ventilation was maintained by use of a $900 \mathrm{C}$ ventilator (Siemens Elema AB). Vascular catheters (arterial and central venous, Swan-Ganz) and a short large-bore cannula in the external jugular vein were inserted through groin and neck incisions, respectively, for blood sampling. After the preparation, the animals rested for $1 \mathrm{~h}$.

Blood pressure was recorded every $5 \mathrm{~min}$ with other physiological measurements [e.g., cardiac output (CO) and pulmonary arterial pressure (PAP)] being made every $30 \mathrm{~min}$. Blood was collected for biochemical analysis and kinin extraction every $15 \mathrm{~min}$.

Blood sampling and extraction for the kinin measurement was based on previously reported procedures $(19,21)$. Briefly, a $10-\mathrm{ml}$ plastic syringe was filled with $3 \mathrm{ml}$ of $0.8 \mathrm{M} \mathrm{HCl}$ and cooled on ice. Through the large-bore cannula in the external jugular vein, $5 \mathrm{ml}$ of blood were drawn into the syringe. Care was taken to achieve maximum aspiration speed by avoiding contact between the tip of the syringe and the vessel wall. During the aspiration the syringe was rotated for the rapid mixing of $\mathrm{HCl}$ and blood. The sample was then injected immediately into a second plastic syringe containing $2 \mathrm{ml}$ of ice-cooled $0.8 \mathrm{~N}$ $\mathrm{HCl}$, shaken vigorously, and placed on ice. The final $\mathrm{pH}$ of the sample was adjusted to 2.0 by the addition of $1.15 \mathrm{ml}$ of a $2 \mathrm{~N}$ $\mathrm{NaOH}$ solution. After $3.68 \mathrm{~g}$ of $\mathrm{NaCl}$ were added to the sample, it was mixed in an ice-cooled kinematic inversion shaker (Turbola, Bachofen, Basel, Switzerland) for $10 \mathrm{~min}$.

For the kinin extraction, $3 \mathrm{ml}$ of $n$-butanol were added to each syringe, and the sample was mixed again for $4 \mathrm{~min}$ and then centrifuged for $4 \mathrm{~min}$ at $1,800 \mathrm{~g}$. The butanol phase was separated into an ice-cooled test tube, and the process was repeated two more times with the centrifugation period extended to 6 and $8 \mathrm{~min}$, respectively. The three butanol fractions were pooled, and the cellular debris and residual plasma proteins were discarded. For the removal of lipids and simultaneous reextraction of kinins into an aqueous medium, $1.5 \mathrm{ml}$ of petroleum ether and $2 \mathrm{ml}$ of distilled water were added to the butanol extracts, and the solution was mixed in the shaker for $10 \mathrm{~min}$ and then centrifuged at $800 \mathrm{~g}$ for $14 \mathrm{~min}$. The aqueous phase was then transferred into Eppendorf tubes with a glass Pasteur pipette, frozen, and stored at $-80^{\circ} \mathrm{C}$ for subsequent assay.
Plasma levels of uncleaved kininogen were measured by allowing trypsin to degrade any high- and low-molecular-mass kininogen contained in the sample, with subsequent measurement of the released kinin $(22,24)$.

The assay of the kinin levels in the blood as well as the measurement of kinin produced from intact kininogen in the plasma (see above) utilized a double-antibody radioimmunoassay (21). The antikinin antibody was from K. Shimamoto (Sapporo, Japan). The second antibody, anti-rabbit globulin from the donkey, was purchased from IDS (Tyne and Wear, UK). The detection limit for kinin using this technique is $30 \mathrm{fmol} / \mathrm{ml}$.

The anaphylatoxin $\mathrm{C} 3 \mathrm{a}$ was measured as des-Arg-C3a, a stable and biologically inactive degradation product of C3a produced by carboxypeptidase B. A modified radioimmunoassay was used (22) based on a method described for human des-ArgC3a (12) with the antiserum against des-Arg-C3a raised in rabbits. des-Arg-C3a and antiserum against des-Arg-C3a were gifts from the late B. Damerau (Göttingen, Germany). The fibrin monomer in plasma was measured with an enzyme immunoassay based on a monoclonal antibody (23). This antibody binds to the $\mathrm{NH}_{2}$-terminal end of the $\alpha$-chain of porcine fibrin. Thrombin cleavage of known amounts of fibrinogen was used to prepare the standard curve used in the assay.

\section{DXS and Hoe-140}

Six weaned domestic pigs with a body weight ranging from 26 to $34 \mathrm{~kg}$ were used in this study. Anesthesia, ventilation, and surgical procedure were the same as described above. All animals received DXS $(2 \mathrm{mg} / \mathrm{kg})$ as a continuous infusion over 60 min. Three animals were randomized to receive a bolus injection of Hoe-140 (30 $\mu \mathrm{g} / \mathrm{kg}$ body wt) $10 \mathrm{~min}$ before the start of the DXS infusion. Hoe-140 was a gift from B. A. Schoelkens and K. J. Wirth, Hoechst AG, Frankfurt, Germany. Three control animals received a saline injection instead of Hoe-140. The dose of Hoe-140 was chosen on the basis of preliminary inhibition experiments with bolus injections of bradykinin as well as pilot experiments with DXS (data not shown). The dose chosen for this study was found to completely block the hypotensive response to bolus injections of bradykinin up to $2,000 \mathrm{ng} / \mathrm{injec}$ tion; the threshold dose needed to produce a decrease of 3-5 $\mathrm{mmHg}$ in diastolic blood pressure was 10-20 $\mathrm{ng}$ of bradykinin before the administration of Hoe-140. CO was measured every 5 min with the thermodilution method; arterial and venous pressures in the systemic and pulmonary circulations were recorded simultaneously. Systemic vascular resistance (SVR) was calculated by using a standard formula $\{79.9$ [mean arterial blood pressure (MABP) - central venous pressure]/CO $\}$. Total protein was measured with the Biuret method.

\section{Statistical Analysis}

Unless otherwise noted, data are presented as means $\pm \mathrm{SE}$. The level of significance was chosen as $\alpha=0.05$.

The evaluation of the blood pressure changes produced by DXS had to take into account that administration of the lower doses of the inhibitor only delayed the time of onset of DXS-induced hypotension and that complete inhibition would occur only at higher doses. Therefore, for each individual experiment, we measured the time after the start of the DXS infusion at which the blood pressure had fallen to $\leq 60 \%$ of the baseline value. These data were analyzed with the Mantel-Haenszel test. The software program that was used (TEST, Institut für Datenanalyse und Versuchsplanung, Gauting, Germany) simultaneously performed multiple Scheffé-like comparisons between the groups.

Furthermore, the distribution of the measurements of uncleaved kininogen was not normal and the baseline levels were not equal in all groups. Here, the time from start of the DXS infusion to a $50 \%$ decrease of the uncleaved kininogen level was 


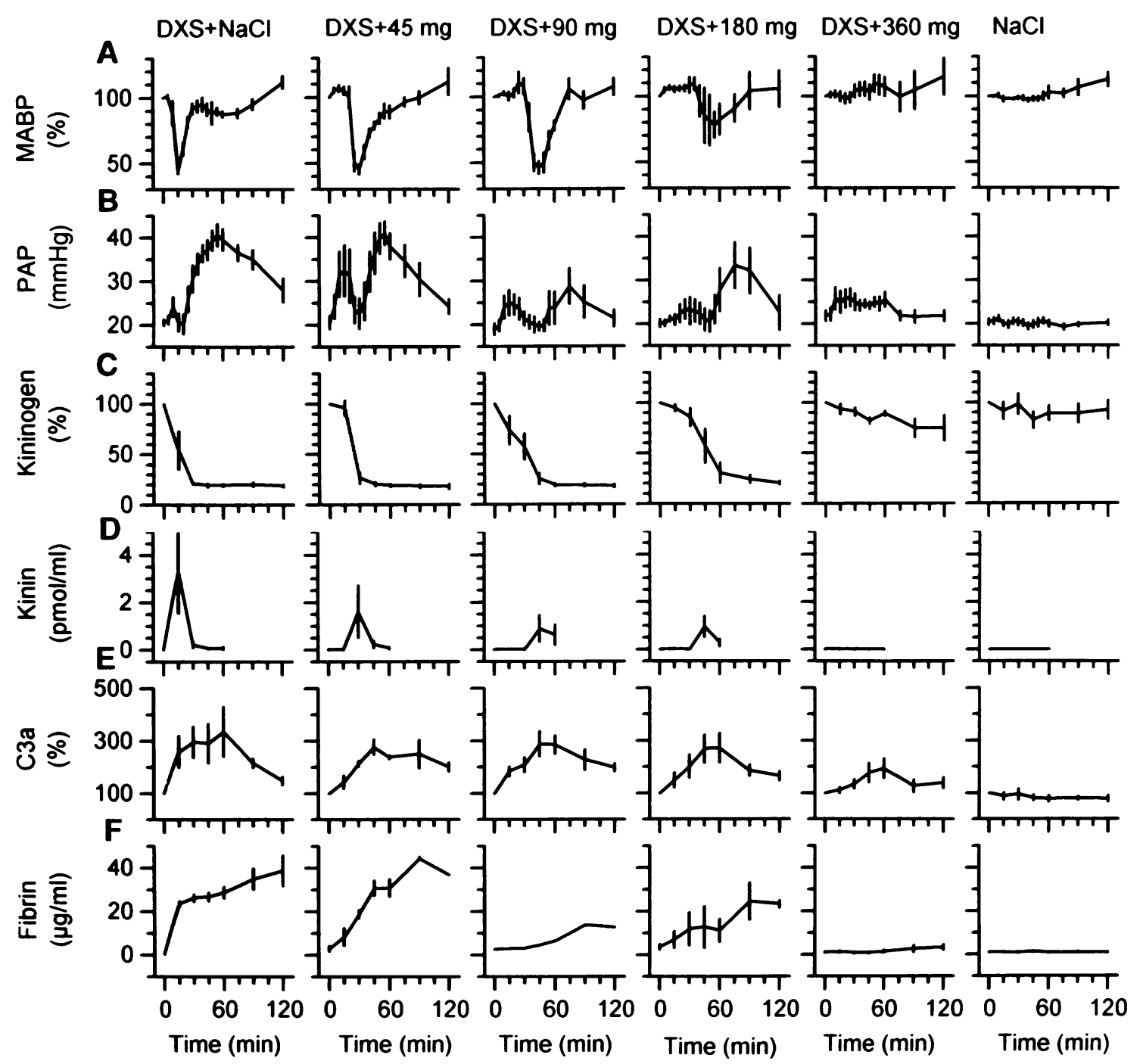

FIG. 1. Effects of dextran sulfate (DXS) and plasma kallikrein inhibitor BAY x 4620 over time. Miniature pigs received DXS infusion ( $5 \mathrm{mg} / \mathrm{kg}$ iv) for $1 \mathrm{~h}$ plus either saline solution or BAY x 4620 in doses of 45, 90, 180, or $360 \mathrm{mg}$ over $2 \mathrm{~h}$ ( 5 groups) or received saline solution alone (1 group). A: early decrease of mean arterial blood pressure (MABP; \% of baseline value) in control group was delayed by low doses of inhibitor, attenuated by higher dose, and completely prevented by highest dose $(P<0.001) . B$ : DXS alone produced biphasic rise in pulmonary arterial pressure $(P A P)$ at 10 and $60 \mathrm{~min}$. Secondary rise in PAP was attenuated by high doses of inhibitor $(P=0.0017)$. $C$ : kinin-containing kininogen in plasma is given as \% of baseline value. Early decrease in kininogen in control group was delayed by low and higher doses of inhibitor and blocked by highest dose $(P<0.001)$. D: release of kinin was attenuated with increasing doses of inhibitor and completely blocked by highest dose $(P<0.0015)$. E: anaphylatoxin des-Arg-C3a $(\mathrm{C} 3 a)$ in plasma is given as \% of baseline value. C3a in plasma rose to reach maximum at $1 \mathrm{~h}$ in control group. Formation of C3a was attenuated with increased doses of inhibitor $(P<0.05)$. $F$ : in group that received DXS alone, fibrin rose substantially. Higher doses of inhibitor produced partial inhibition, with highest dose producing complete inhibition of fibrin formation $(P<0.05)$.

measured in each animal and analyzed with the aforementioned procedure (Mantel-Haenszel test).

The distribution of the measurements of kinin also was not normal, but, in this case, the Kruskall-Wallis analysis of ranks was used. For a global test, the average of all repeated measurements during DXS infusion in each animal was formed. Multiple tests at each time were decided according to the closure principle (16).

For a global test of PAP, the average of the repeated measurements during DXS infusion in each animal was formed. These values were analyzed by analysis of covariance, with the baseline measurement as covariate. Multiple comparisons between groups were made with Tukey's test. Because of their distribution, results of des-Arg-C3a and fibrin were analyzed with Kruskall-Wallis analysis. A global value could not be formed because of missing values. Therefore, the multiple niveau alpha was ascertained with a modified Bonferroni procedure (10). For the data involving DXS and Hoe-140, the statistical analysis was limited to a comparison at $25 \mathrm{~min}$, the time of maximum blood pressure decrease. The Mann-Whitney $U$ test was used with $P<0.05$ considered significant.

\section{RESULTS}

\section{$D X S$ and $B A Y \times 4620$}

$M A B P$ (Fig. 1). DXS (5 mg/kg) induced a rapid decline in MABP that reached a nadir of $44.5 \pm 2.6 \%$ of baseline value at $15 \mathrm{~min}$ in the group $\mathrm{DXS}+\mathrm{NaCl}$. MABP returned to normal levels by 30 min after the DXS infusion was started. In the group that received DXS and the lowest dose of BAY x 4620 (DXS + 45 mg of BAY x 4620), 

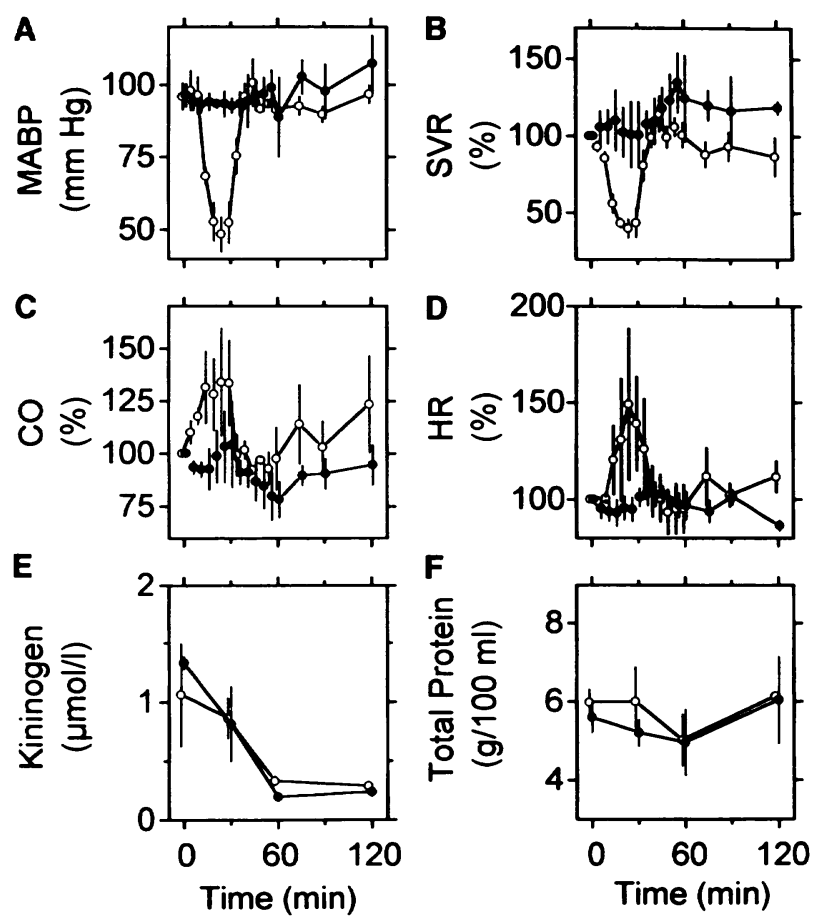

FIG. 2. Effects of DXS and Hoe-140 over time. Six weaned pigs received DXS infusion iv ( $2 \mathrm{mg} / \mathrm{kg}$ ) for $1 \mathrm{~h}$. In addition, 3 pigs received 30 $\mu \mathrm{g} / \mathrm{kg}$ of Hoe-140 (๑) $15 \mathrm{~min}$ before DXS and 3 pigs received saline solution $(O)$. Time course in each group is presented as mean $\pm \mathrm{SE}$. $x$-Axis, time after onset of DXS infusion. $A$ : control animals had sharp decrease in MABP from 15 to 35 min after onset of DXS infusion, whereas Hoe-140-treated animals had completely stable blood pressure; their MABP was significantly higher at $25 \mathrm{~min}$. $B$ : systemic vascular resistance (SVR) is given as \% of baseline value. Arterial hypotension in control animals was paralleled by systemic vasodilation, whereas Hoe-140-treated animals had stable SVR; their SVR was significantly higher at $25 \mathrm{~min}$. $C$ : cardiac output $(\mathrm{CO})$ is given as \% of baseline value. From 5 to 30 min after onset of DXS infusion, $\mathrm{CO}$ was elevated in control animals. Hoe-140-treated animals had unchanged CO (NS). $D$ : heart rate (HR) is given as \% of baseline value. Control animals responded with rise in HR to DXS infusion. Hoe-140-treated animals had no change in HR (NS). $E$ : values of uncleaved kininogen declined to $18 \pm 1 \%$ of baseline without any difference between groups. $F$ : values of total protein remained stable.

MABP remained stable until $20 \mathrm{~min}$, decreased to its nadir of $45 \pm 3.5 \%$ after $30 \mathrm{~min}$, and then returned to baseline level. The augmented dose of the inhibitor (DXS $+90 \mathrm{mg}$ of BAY $x 4620$ ) led to a further delay in the onset of hypotension that reached its nadir of $46 \pm 4.8 \%$ at 45 min. The next inhibitor dose level (DXS +180 of $\mathrm{mg}$ BAY x 4620) not only delayed the onset but also reduced the magnitude of hypotension to $78 \pm 10 \%$ at $55 \mathrm{~min}$. MABP remained constant during the entire observation period in the group with DXS and the highest inhibitor dose (DXS + $360 \mathrm{mg}$ of BAY $\times$ 4620) and in the control group without DXS. The time to $60 \%$ hypotension differed significantly between the groups (Mantel-Haenszel test, $P<0.001$ ), and in the multiple comparison test, the difference between DXS $+\mathrm{NaCl}$ and DXS $+360 \mathrm{mg}$ of BAY x 4620 was statistically significant $(P<0.001)$.

$P A P$ (Fig. 1). PAP displayed a biphasic rise to $118 \pm$ $11 \%$ at $10 \mathrm{~min}$ and $198 \pm 13 \%$ at $55 \mathrm{~min}$ in response to the DXS infusion. Administration of BAY $x 4620$ delayed or attenuated only the second PAP increase, with the group receiving DXS $+360 \mathrm{mg}$ of BAY $\times 4620$ showing an early rise to $120 \%$ of baseline value but no secondary peak. The groups were significantly different in the global test (mean value during DXS infusion, analysis of covariance, $P=0.0017$ ), but multiple between-group comparisons did not reveal a significant difference between DXS $+\mathrm{NaCl}$ and DXS $+360 \mathrm{mg}$ of BAY x 4620 . All multiple tests over time were significant from 35 to $90 \mathrm{~min}$.

Circulating kininogen (Fig. 1). In the group DXS + $\mathrm{NaCl}$, uncleaved kininogen in plasma decreased to $54 \pm$ $19 \%$ at $15 \mathrm{~min}$ and to $21 \pm 1 \%$ at $30 \mathrm{~min}$ and remained around $20 \pm 1 \%$ of baseline value for the remainder of the study. This decrease in uncleaved kininogen was delayed by the simultaneous administration of BAY x 4620; in the group DXS $+180 \mathrm{mg}$ of BAY $\times 4620$, kininogen decreased to $30 \pm 10 \%$ at $60 \mathrm{~min}$. In the group with the highest dose of the inhibitor, DXS $+360 \mathrm{mg}$ of BAY $\mathrm{x}$ 4620 , and in the group without DXS, no decrease in kininogen was observed. The time to $50 \%$ kininogen decrease differed significantly between the groups (Mantel-Haenszel test, $P<0.001$ ). The difference between $\mathrm{DXS}+\mathrm{NaCl}$ and DXS $+360 \mathrm{mg}$ of BAY $\times 1620$ was statistically significant (multiple Scheffé-like comparisons, $P<0.02)$.

Free kinin (Fig. 1). Kinin levels in blood as a function of time corresponded closely with the changes seen in MABP. BAY x 4620 produced a dose-dependant delay in the release of kinin with increasing doses while also progressively reducing the amplitude of kinin release. In the group with the highest inhibitor dose, DXS $+360 \mathrm{mg}$ of BAY x 4620, only two of five animals had kinin levels above the detection limit, the highest being $47 \mathrm{fmol} / \mathrm{ml}$. The group without DXS had no detectable kinin release. The global test as well as all multiple tests for repeated measurements during the infusion of DXS revealed a statistically significant difference between the groups $(P$ $<0.002$ ). The comparison between the groups DXS + $\mathrm{NaCl}$ and $\mathrm{DXS}+360 \mathrm{mg}$ of BAY x 4620 was significant $(P<0.02)$.

Complement activation (Fig. 1). The plasma concentration of des-Arg-C3a rose to three times baseline value at $60 \mathrm{~min}$ in the group DXS $+\mathrm{NaCl}$. All groups except the control group without DXS showed such an increase, with a peak at $60 \mathrm{~min}$ followed by a slow decline. The peak of des-Arg-C3a flattened when the dose of the inhibitor increased. Except for baseline measurements, at each time the difference between the groups was statistically significant, and the global test was significant (Hommel's multiple test, $P<0.05$ ).

Fibrin monomer (Fig. 1). Fibrin monomer in plasma increased steadily during the 2 -h observation period, with peak levels of $39 \pm 7 \mu \mathrm{g} / \mathrm{ml}$ being reached at $2 \mathrm{~h}$ in the group DXS $+\mathrm{NaCl}$. Except for the control group without DXS administration, all groups had an increase in fibrin monomer that showed a dose-dependent reduction based on the amount of inhibitor. The difference between the groups was statistically significant at each time and in the global test $(P<0.05)$. It must be noted, however, that at the baseline value there was a marginal difference between the groups $(P=0.051)$.

\section{DXS and Hoe-140 (Fig. 2)}

The control animals in this study experienced severe arterial hypotension similar to that seen in Fig. $1 A$. DXS 
$(2 \mathrm{mg} / \mathrm{kg})$ induced a rapid decline in MABP that reached a nadir of $51 \pm 8 \%$ of baseline value at $25 \mathrm{~min}$. MABP returned to baseline values at $40 \mathrm{~min}$. In the Hoe-140treated group MABP remained completely stable. These groups were significantly different $(P<0.05)$.

The arterial hypotension of the control animals was paralleled by a decrease in SVR. SVR decreased to $40 \pm$ $6 \%$ at $25 \mathrm{~min}$ and was restored to baseline levels at 40 min. The Hoe-140-treated group did not experience this vasodilation, with the difference being statistically significant $(P<0.05)$.

Hypotension and vasodilation were accompanied by rises in cardiac output and heart rate (HR). In the control animals, CO rose to $134 \pm 25 \%$ at $25 \mathrm{~min}$ and returned to baseline values at $40 \mathrm{~min}$, and HR rose to $150 \pm$ $40 \%$ at $25 \mathrm{~min}$ and returned to baseline values at $45 \mathrm{~min}$. Both effects appeared to be prevented in the Hoe-140treated group, but the differences were not statistically significant.

Uncleaved kininogen decreased to $18 \%$ with no apparent difference between the groups, whereas total protein remained stable in both groups.

\section{DISCUSSION}

The principal finding in this study was that both BAY $x 4620$, a plasma kallikrein inhibitor, and Hoe-140, a bradykinin $\mathrm{B}_{2}$-receptor antagonist, prevented DXS-induced hypotension in pigs.

DXS infusion produced all the effects that would be predicted, assuming that it is an activator of the contact system. At $15 \mathrm{~min}$, very high kinin concentrations in blood were found, which closely correlated with a brisk decrease in arterial blood pressure. Uncleaved kininogen decreased and reached a steady-state level at $30 \mathrm{~min}$, whereas anaphylatoxin $\mathrm{C} 3 \mathrm{a}$ increased, reaching a peak at $60 \mathrm{~min}$. Fibrin monomer also increased continuously during the observation period. These data corroborate our assumption that activation of the contact system had taken place as a consequence of the infusion of DXS.

DXS infusion also produced alterations in lung function, particularly a transient increase in mean PAP. Other parameters of lung function, such as extravascular lung water and airway pressure, however, showed only minor changes (data not shown). One possible explanation for the increase in PAP is the activation of thrombin because thrombin is a potent pulmonary vasoconstrictor (11). However, our data do not rule out other causes of the PAP rise.

Administration of BAY $\times 4620$ before and during DXS infusion delayed or attenuated the DXS-induced changes, with the highest dose producing a complete blockade of the DXS-induced changes measured in this system.

Compared with natural aprotinin from bovine lungs, BAY x 4620, the mutant that was employed in our study, is a relatively potent and selective plasma kallikrein inhibitor with an inhibitor constant for human plasma kallikrein roughly 100 -fold lower than that of natural aprotinin. The inhibitor constants of BAY x 4620 for human plasma kallikrein and human plasmin are $5 \times 10^{-10}$ and 2 $\times 10^{-10} \mathrm{M}$, respectively, whereas the corresponding val- ues of wild-type aprotinin are $3 \times 10^{-8}$ and $8 \times 10^{-11} \mathrm{M}$ (W. Schröder, Bayer AG Pharmaceutical Research, Wuppertal, Germany, personal communication).

In this regard, it is important to note that plasma levels of BAY x 4620 were in the range of $1.5 \mu \mathrm{M}$ when a complete blockade of DXS-induced changes was achieved (data not shown). In previous experiments, a complete blockade of DXS-induced hypotension was only achieved with plasma concentrations of wild-type aprotinin $\sim 12.5 \mu \mathrm{M}(9)$. This indicates that BAY $\times 4620$ is also a far better inhibitor of plasma kallikrein than natural aprotinin when used in vivo. As a result, this compound may be useful as a probe to investigate pathological conditions, such as hypotension and bleeding during extracorporeal circulation and hemodialysis and hereditary angioedema, that are based on activation of the contact system.

The parallels between in vitro and in vivo potencies of the two inhibitors aprotinin and BAY $x 4620$ support the notion that DXS-induced hypotension was mediated by bradykinin, a peptide that is released after the specific cleavage of high-molecular-weight kininogen by plasma kallikrein.

Bradykinin is one of the most potent hypotensive agents yet described. The finding that the effect of DXS infusion, namely hypotension, elevated kinin levels in the blood and that the decrease of the precursor of bradykinin, uncleaved kininogen, was prevented by the plasma kallikrein inhibitor BAY x 4620 gives support to the notion that the decrease in blood pressure was mediated by the release of bradykinin. However, other vasoactive mediators such as prostacyclin, platelet-activating factor, or serotonin can be released either directly by DXS or indirectly via thrombin or bradykinin and could also be responsible for all or part of the response. We therefore wanted to determine whether the blockade of bradykinin $\mathrm{B}_{2}$ receptors alone would prevent DXS-induced hypotension.

In the second part of this study, we studied the effect of the $\mathrm{B}_{2}$-receptor antagonist Hoe-140 on DXS-induced hypotension. In this study, DXS infusion also led transiently to severe arterial hypotension that was accompanied by vasodilation and increases in $\mathrm{HR}$ and $\mathrm{CO}$ in the control animals, whereas Hoe-140 completely blocked these effects in the treated group. However, serial measurements of uncleaved kininogen demonstrate that effective activation of the contact system had taken place equally in both groups. Total protein measurements were unchanged in both groups, making specific cleavage the likely cause for the decrease in kininogen. The relatively small sample size in this study was selected on the basis of pilot experiments that indicated that the actual dose of Hoe-140 used in this study would completely block bradykinin-induced hypotension. The finding that the bradykinin receptor blockade with Hoe-140 completely abolished DXS-induced hypotension once more points to bradykinin as the primary causative mediator.

In summary, we have shown that DXS infusion in pigs produces activation of the contact system of blood coagulation with the following consequences: the appearance of high kinin levels in blood; a decrease in uncleaved kininogen in plasma; severe transient arterial hypotension 
accompanied by vasodilation and a compensatory rise in $\mathrm{CO}$ and HR; and activation of the coagulation, complement, and fibrinolysis cascades. Both the plasma kallikrein inhibitor BAY $\times 4620$ and the bradykinin $\mathrm{B}_{2}$ receptor antagonist Hoe-140 were able to block DXSinduced hypotension. Only the former compound prevented the decrease of uncleaved kininogen, whereas the latter compound prevented hypotension during ongoing kininogen cleavage. We conclude that DXS infusion led to the release of bradykinin from kininogen via activation of the contact system and led to arterial hypotension via stimulation of the $B_{2}$ kinin receptor.

Astrid Braune and Tanja Hennes provided skillful technical assistance.

Part of this work was supported by the Deutsche Forschungsgemeinschaft (SFB 207).

Address for reprint requests: M. Siebeck, Dept. of Surgery, Univ. of Munich, Klinikum Innenstadt, Nussbaumstrasse 20, D-80336 Munich, Germany.

Received 31 January 1994; accepted in final form 28 July 1994.

\section{REFERENCES}

1. Binnema, D. J., G. Dooijewaard, J. J. L. van Iersel, P. N. C. Turion, and C. Kluft. The contact-system dependent plasminogen activator from human plasma: identification and characterization. Thromb. Haemostasis 64: 390-397, 1990.

2. Cochrane, C. G., and S. D. Revak. Dissemination of contact activation in plasma by plasma kallikrein. J. Exp. Med. 152: 608$619,1980$.

3. Colman, R. W. Activation of plasminogen by human plasma kallikrein. Biochem. Biophys. Res. Commun. 35: 273-279, 1969.

4. Colman, R. W., D. N. Flores, R. A. de la Cadena, C. F. Scott, L. Cousens, P. J. Barr, I. B. Hoffman, F. Kueppers, D. Fisher, S. Idell, and J. Pisarello. Recombinant alpha 1-antitrypsin Pittsburgh attenuates experimental gram-negative septicemia. Am. J. Pathol. 130: 418-426, 1988.

5. De la Cadena, R. A., A. F. Suffredini, J. D. Page, R. A. Pixley, N. Kaufman, J. E. Parrillo, and R. W. Colman. Activation of the kallikrein-kinin system after endotoxin administration to normal human volunteers. Blood 81: 3313-3317, 1993.

6. DiScipio, R. G. The activation of the alternative pathway $\mathrm{C} 3 \mathrm{con}$ vertase by human plasma kallikrein. Immunology 45: 587-595, 1982.

7. Ebbers, J., D. Hörlein, M. Schedel, and R. Das (Inventors). Recombinant Aprotinin Variants Genetically Engineered Process for the Microbial Preparation of Homogeneously Processed Aprotinin Variants and the Therapeutic Use Thereof. US Patent 5,164,482. 17 Nov. 1992. Int. Cl. CO7K 15/04, CO7K 15/06, CO7K 7/10.

8. Ghebrehiwet, B., B. P. Randazzo, J. T. Dunn, M. Silverberg, and A.P. Kaplan. Mechanisms of activation of the classical pathway of complement by Hageman factor fragment. J. Clin. Invest. 71: 1450-1456, 1983.

9. Hoffmann, H., M. Siebeck, O. Thetter, E. Fink, and A. Phi- lapitsch. Effect of aprotinin and C1-esterase inhibitor on activation of the plasma kallikrein-kinin system in vivo. Prog. Clin. Biol. Res. 236A: 159-164, 1987.

10. Hommel, G. A stagewise rejective multiple test procedure based on a modified Bonferroni test. Biometrika 75: 383-386, 1988.

11. Horgan, M. J., J. W. Fenton, and A. B. Malik. $\alpha$-Thrombin-induced pulmonary vasoconstriction. J. Appl. Physiol. 63: 1993-2000, 1987.

12. Hugli, T., and D. Chenoweth. Biologically active peptides of complement - techniques and significance of $\mathrm{C} 3 \mathrm{a}$ and $\mathrm{C} 5 \mathrm{a}$ measurements. In: Immunoassays: Clinical Laboratory Techniques for the 1980s, edited by R. Nakamura, S. Dito, and E. Tucker. New York: Liss, 1980, p. 443-460.

13. Hugli, T. E., and H. J. Müller-Eberhard. Anaphylatoxins: C3a and C5a. Adv. Immunol. 26: 1-53, 1978.

14. Kalter, E. S., M. R. Daha, J. W. ten Cate, J. Verhoef, and B. N. Bouma. Activation and inhibition of Hageman factor-dependent pathways and the complement system in uncomplicated bacteremia or bacterial shock. J. Infect. Dis. 151: 1019-1027, 1985.

15. Kluft, C. Determination of prekallikrein in human plasma-optimal conditions for activating prekallikrein. J. Lab. Clin. Med. 91: 83-95, 1978.

16. Marcus, R., E. Peritz, and K. R. Gabriel. On closed testing procedures with special reference to ordered analysis of variance. Biometrika 63: 655-660, 1976.

17. McGuire, W. W., R. G. Spragg, A. B. Cohen, and C. G. Cochrane. Studies on the pathogenesis of the adult respiratory distress syndrome. J. Clin. Invest. 69: 543-553, 1982.

18. Morrison, D. C., and C. G. Cochrane. Direct evidence for Hageman factor (factor XII) activation by bacterial lipopolysaccharides (endotoxins). J. Exp. Med. 140: 797-810, 1974.

19. Rett, K., M. Wicklmayr, E. Fink, E. Maerker, G. Dietze, and H. Mehnert. Local generation of kinins in working skeletal muscle tissue in man. Biol. Chem. Hoppe-Seyler 370: 445-449, 1989.

20. Rhaleb, N., N. Rouissi, D. Jukic, D. Regoli, S. Henke, G. Breipohl, and J. Knolle. Pharmacological characterization of a new highly potent B2 receptor antagonist (Hoe-140: D$\mathrm{Arg}^{0}\left[\mathrm{Hyp}^{3}, \mathrm{Thi}^{5}, \mathrm{D}-\mathrm{Tic}^{7}, \mathrm{Oic}^{8}\right]$-bradykinin). Eur. J. Pharmacol. 210: 115-120, 1992.

21. Shimamoto, K., T. Ando, S. Tanaka, Y. Nakahashi, T. Nishitani, S. Hosoda, H. Ishida, and O. Iimura. An improved method for the determination of human blood kinin levels by sensitive kinin radioimmunoassay. Endocrinol. Jpn. 29: 487-494, 1982.

22. Siebeck, M., E. Fink, J. Weipert, M. Jochum, H. Fritz, M. Spannagl, P. Kroworsch, K. Shimamoto, and L. Schweiberer. Inhibition of plasma kallikrein with aprotinin in porcine endotoxin shock. J. Trauma 34: 193-198, 1993.

23. Spannagl, M., A. Trauner, A. Birg, G. Frank, H. Hoffmann, M. Siebeck, and H. Lill. Sensitive detection of the activation state of blood coagulation in porcine DIC models by a new fibrin immunoassay. Blood Coag. Fibrinol. 4: 103-106, 1993.

24. Uchida, Y., and M. Katori. Differential assay method for high molecular weight and low molecular weight kininogens. Thromb. Res. 15: 127-134, 1979.

25. Wiggins, R. C., A. Glatfelter, A. M. Campbell, R. G. Kunkel, and R. J. Ulevitch. Acute hypotension due to platelet serotonininduced chemoreflexes after intravenous injection of dextran sulfate in the rabbit. Circ. Res. 57: 262-277, 1985. 